\section{Prosthetic valve endocarditis caused by Pasteurella in a penicillin allergic patient: challenges in diagnosis and treatment}

\author{
Giovanni Satta, Rebecca Louise Gorton, \\ Hala Kandil \\ Department of Medical Microbiology, \\ Royal Free London NHS Foundation \\ Trust, London, UK
}

\begin{abstract}
Pasteurella multocida is a rare cause of infective endocarditis with only a few cases described. This report involves a 38-year-old penicillin-allergic patient in an immunocompromised state with several co-morbidities. Two molecular microbiological techniques, $16 \mathrm{~S}$ rRNA sequencing and matrix-assisted laser desorption/ionization time-of-flight mass spectrometry were used to confirm the species identification as $P$. multocida. Previous reports in the literature are also reviewed.
\end{abstract}

\section{Introduction}

The gram-negative coccobacillus Paste urella multocida is normally found in the oral cavity of dogs and cats. It is a recognised cause of wound infection as a result of bites, licking or scratching. Bacteraemia and endocarditis are uncommon complications and, from literature reviews, are found to be associated with prior animal trauma, a second source of infection (e.g. cellulitis) and high mortality rates. ${ }^{1}$ We herein report a case in which a pet owner developed $P$. multocida endocarditis and was subsequently treated via OPAT (outpatient parenteral antimicrobia therapy) service with an excellent clinical outcome. Species identification of $P$. multoci$d a$ was performed using traditional microbiological methods and confirmed with $16 \mathrm{~S}$ rRNA sequencing and matrix-assisted laser desorption/ionization time-of-flight mass spectrometry (MALDI-TOF).

\section{Brief Report}

A 38-year-old man was admitted to our hospital with a 2 days history of fever, malaise and cellulitis around a Mitrofanoff abdominal catheter. Multiple comorbidities included
Marfan syndrome (with metallic aortic valve inserted sixteen years before) and congenital urological abnormalities that led to the Mitrofanoff appendicovesicostomy and a recent renal transplant, five years and six months before admission respectively. He was on steroids and tacrolimus as immunosuppressive therapy.

On presentation, the patient was febrile, hypotensive and tachycardic, had a normal leukocyte count $\left(5.09 \times 10^{9} / \mathrm{L}\right)$ but raised Creactive protein (CRP) $45 \mathrm{mg} / \mathrm{L}$ with mild anaemia ( $\mathrm{Hb}$ of $10.1 \mathrm{~g} / \mathrm{dL}$ from a baseline of $14.6 \mathrm{~g} / \mathrm{dL}$ ) and renal impairment (creatinine $163 \mathrm{umol} / \mathrm{L}$ and urea $8.1 \mathrm{mmol} / \mathrm{L}$ ). The cellulitis around the Mitrofanoff abdominal catheter was noted and a swab was taken for culture. Urine and blood cultures were also taken and sent to microbiology. His chest X-ray did not show any relevant consolidation and abdominal ultrasound noted two small abdominal collections that had already been noted posttransplant and had since decreased in size. The patient was documented to be penicillin allergic (previous skin rash), so was initiated on Ertapenem IV $1 \mathrm{gr} / 0 \mathrm{D}$. The cellulitis improved and the patient was clinically well within 48 hours of commencing antibiotics.

The blood culture set (aerobic and anaerobic bottles) taken on admission was positive with $P$. multocida but the abdominal swab grew only mixed anaerobes. Urine culture was negative and all subsequent blood cultures taken after starting Ertapenem were negative after 5 days of incubation.

Transesophageal (TEE) echocardiography revealed the presence of a vegetation $(0.57 \times$ $0.2 \mathrm{~cm}$ ) above the atrium of the right coronary artery. A previous transthoracic echocardiogram (TTE), around one month prior to admission, was reported as negative. Therefore, the patient was treated following our local protocol for infective endocarditis: he was switched to Ceftriaxone IV $2 \mathrm{gr} / 0 \mathrm{D}$, a PICC line was inserted in the right arm and he was discharged under the OPAT (outpatient parenteral antimicrobial therapy) service to complete six weeks IV antibiotics as an outpatient. On follow-up, two repeat TTEs showed clear aortic valve.

\section{Discussion}

We reviewed all the previous reported cases of $P$. multocida endocarditis in the literature through PubMed and cross-references search. To our knowledge this is the twentyfirst reported case of endocarditis but the first to be reported in a penicillin allergic patient.${ }^{10}$ As with previous cases, the diagnosis of infective endocarditis was made according to Duke criteria: one major criterion (evidence
Correspondence: Giovanni Satta, Department of Medical Microbiology, Royal Free London NHS Foundation Trust, Pond St, London, NW3 2QG, UK. Tel: +044.0.207794 0500 - Fax +44.0.207317 7726. E-mail: giovanni.satta@nhs.net

Key words: Pasteurella multocida, endocarditis, 16S-rRNA, MALDI-TOF.

Conflict of interests: the authors report no conflict of interest.

Received for publication: 13 November 2011. Revision received: 22 February 2012.

Accepted for publication: 13 April 2012.

This work is licensed under a Creative Commons Attribution NonCommercial 3.0 License (CC BYNC 3.0).

(C) Copyright G. Satta et al., 2012

Licensee PAGEPress, Italy

Infectious Disease Reports 2012; 4:e32

doi:10.4081/idr.2012.e32

of endocardial involvement) and three minor criteria (predisposing heart condition, fever and microbiological evidence with positive blood culture). Laboratory isolation and identification of $P$. multocida was performed using standard microbiological techniques: API20NE, API-20E (BioMerieux) and the Phoenix Automated System (BD). Interestingly, both API profiles reported a low confidence value for identification as $P$. multocida to the species level (CV 53\%) and the Phoenix assay only identified the isolate to genus level as Pasteurella spp. Thus, confirmation of species identification was performed using $16 \mathrm{~S}$ rRNA sequencing and MALDI-TOF protein profiling: 16S rRNA sequence was performed using PCR parameters detailed in Maskell NA et al. (2006) and we obtained a 584bp fragment sharing $99 \%$ sequence homology to that of $P$. multocida sub species septica. MALDI-TOF profiling was performed using a Bruker Daltonics Microflex platform with recommended $\alpha$-Cyano-4-hydroxycinnamic acid matrix under pre-defined conditions as specified by Bruker. The MALDI-TOF mass spectra for the clinical isolate was analysed against MALDI-TOF mass spectra for 3,438 species of bacteria located in the Bruker Daltonics flexAnalysis 3.0 database. The clinical isolate was identified as $P$. multocida with a confidence value of 2.321 indicating highly probable species identification, with no differential bacterial species being reported. Sensitivities were as follow (EUCAST breakpoints): penicillin MIC (mg/L) was 0.19 , ciprofloxacin 0.016 , doxycycline 0.5 , co-trimoxazole 0.032 , ertapenem 0.016 and Ceftriaxone $<0.016$, all being susceptible. Of note the Gentamicin 
MIC (mg/L) was $2 \mathrm{mg} / \mathrm{L}$ with a susceptibility breakpoint of $2 \mathrm{mg} / \mathrm{L}$. It is already known that Pasteurella can be easily misindentified. The accuracy of previous reports in the literature has been questioned as their standard microbiological description was incomplete and Pasteurella species may be misidentified as Haemophilus spp., which are well known causes of endocarditis. They both belong to the Pasteurellaceae family and based on the genomic sequence it appears they diverged approximately 270 million years ago. ${ }^{11}$

Pasteurella spp. are Gram-negative coccobacilli generally present in the oral cavity of cats and dogs. To date twenty-one species have been identified and the species $P$. multocida can be further divided into three different subspecies (subspecies multocida, septicum and gallicida). The spectrum of infections varies from skin and soft tissue to central nervous system infections. ${ }^{1}$ Ten out of twenty-one (47.6\%) endocarditis cases described in the literature had close contact with animals detailed in the clinical history.${ }^{10}$ Our patient did not have a clear history of bites or scratches but he did have two cats at home and close contact with dogs at his sister's house. He was also receiving immunosuppressive therapy after his renal transplant. Other known risk factors include cirrhosis and chronic respiratory illness. In fact, of the cases of $P$. multocida endocarditis reviewed, two out of twenty-one (9.5\%) had cirrhosis as underlying condition.

Penicillin still remains the best antimicrobial agent for the treatment of virtually all forms of Pasteurella infection. A fluoroquinolone, doxycycline, or co-trimoxazole should be considered for skin and soft tissue infections as an alternative for patients with intolerance to $\beta$-lactams. There are no clear guidelines about the treatment of $P$. multoci$d a$ endocarditis in penicillin-allergic patients. Our choice was based on the susceptibility results. The patient was known to be penicillin allergic (previous rash) and ciprofloxacin and doxycycline were contraindicated as causing interaction with his anticoagulant treatment. We decided then to switch to Ceftriaxone as this antibiotic is already vastly used in the treatment of endocarditis caused by other Gram-negative organisms and is more cost effective. We did not consider necessary to treat with a combination therapy. Aminoglycosides have moderate to poor in vitro activity against Pasteurella and probably should not be used, particularly given the paucity of clinical experience. ${ }^{1}$

Finally, our patient improved within 48 hours of commencing antimicrobial therapy and surgical intervention was unnecessary. From the literature, only three other cases have been successfully treated with solely antibiotics and valve replacement was mandatory in seventeen out of twenty-one (81\%) of cases described. ${ }^{2-10}$

\section{Conclusions}

In conclusion, $P$. multocida endocarditis remains a rare infection. Clinicians may face various challenges, from achieving the correct species identification to chosing treatment regimens in penicillin-allergic patients. In our case new diagnostic techniques such as $16 \mathrm{~S}$ rRNA sequencing and MALDI-TOF were demonstrated to be invaluable tools for species confirmation. We propose that MALDI-TOF is a rapid, accurate method for the identification of $P$. multocida from bacterial cultures and can significantly reduce the time to diagnosis of $P$. multocida bacteraemia. Furthermore we have shown that cephalosporins and carbapenems are probably good substitutes for penicillin in case of allergy.

\section{References}

1. Zurlo JJ. Pasteurella Species. In: Mandell GL, Bennett JE, Dolin R. Mandell, Douglas, and Bennett's Principles and Practice of Infectious Diseases, 7th Ed. Philadelphia, PA: Churchhill Livingston/Elsevier; 2009. pp 2939-2942.

2. Singh CP, Spurrell JR. Pasteurella multocida endocarditis. Br Med J 1983;286:1862-3.

3. Hombal SM, Dincsoy HP. Pasteurella multocida endocarditis. Am J Clin Pathol 1992;98: 565-8.

4. Nettles RE, Sexton DJ. Pasteurella multocida prosthetic valve endocarditis: case report and review. Clin Infect Dis 1997;25:920-1.

5. Fukumoto Y, Moriyama Y, Iguro Y, et al. Pasteurella multocida endocarditis: report of a case. Surg Today 2002;32:513-5.

6. Fayad G, Modine T, Mokhtari S, et al. Pasteurella multocida aortic valve endocarditis: case report and literature review. J Heart Valve Dis 2003;12:261-3.

7. Al-Ghonaim MA, Abba AA, Al-Nozha M. Endocarditis caused by Pasteurella multocida. Ann Saudi Med 2006;26:147-9.

8. Graf S, Binder T, Heger M, et al. Isolated endocarditis of the pulmonary valve caused by Pasteurella multocida. Infection 2007;35:43-5.

9. Reinsch N, Plicht B, Lind A, et al.. Recurrent infective endocarditis with uncommon Gram-negative Pasteurella multocida and Pseudomonas aeruginosa: a case report. J Heart Valve Dis 2008;17: 710-3.

10. Naba MR, Araj GF, Kanafani ZA, et al.. First case of Pasteurella multocida endocarditis of the tricuspid valve: a favorable outcome following medical treatment. Int $\mathrm{J}$ Infect Dis 2009;13:e267-9.

11. Bakkali M, Chen TY, Lee HC, et al. Evolutionary stability of DNA uptake signal sequences in the Pasteurellaceae. Proc Natl Acad Sci USA 2004;101:4513-8. 